\title{
Primary Serous Cystadenocarcinoma of Broad Ligament: A Case Report with Laparoscopic, Histopathologic and Immunohistochemical Findings
}

\author{
Kenji Niwa1 ${ }^{*}$, Motoki Takenaka², Takeaki Saitake2, Tiger Koike' ${ }^{2}$, Kentaro Nagata1, \\ Kentaro Niwa ${ }^{3}$, Kohjiro Niwa ${ }^{3}$, Sakae Mori ${ }^{4}$, Keigo Kuwabara ${ }^{4}$, Akane Onogi ${ }^{5}$, \\ Takuji Tanaka ${ }^{5}$
}

\author{
${ }^{1}$ Department of Obstetrics \& Gynecology, Gujo City Hospital, Gujo, Japan \\ ${ }^{2}$ Department of Obstetrics \& Gynecology, Gifu University School of Medicine, Gifu, Japan \\ ${ }^{3}$ Department of Obstetrics \& Gynecology, Kizawa Memorial Hospital, Minokamo, Japan \\ ${ }^{4}$ Section of Laboratory Medicine and Obstetrics \& Gynecology, Gujo City Hospital, Gujo, Japan \\ ${ }^{5}$ Department of Diagnostic Pathology \& Research Center of Diagnostic Pathology, Gifu Municipal Hospital, Gifu, Japan \\ Email: *kniwa.gujo913@gmail.com
}

\begin{abstract}
How to cite this paper: Niwa, K., Takenaka, M., Saitake, T., Koike, T., Nagata, K., Niwa, K., Niwa, K., Mori, S., Kuwabara, K., Onogi, A. and Tanaka, T. (2022) Primary Serous Cystadenocarcinoma of Broad Ligament: A Case Report with Laparoscopic, Histopathologic and Immunohistochemical Findings. Open Journal of Pathology, 12, 22-30.

https://doi.org/10.4236/ojpathology.2022.1 $\underline{21003}$
\end{abstract}

Received: October 20, 2021

Accepted: December 10, 2021

Published: December 13, 2021

Copyright $\odot 2022$ by author(s) and Scientific Research Publishing Inc. This work is licensed under the Creative Commons Attribution International License (CC BY 4.0).

http://creativecommons.org/licenses/by/4.0/

\section{Open Access}

\begin{abstract}
An 86-year-old Japanese woman underwent an examining laparoscopy for removing the huge pelvic tumor. At laparoscope examination, the cystic tumor was found within the left broad ligament, while the ovaries, fallopian tubes and uterus showed almost normal appearance. The tumor was removed together by total laparoscopic hysterectomy and bilateral salpingo-oophorectomies after the suction of serous content in the broad ligament. Cytological findings of the ascites suggested serous carcinoma. The resected ovaries and fallopian tubes were grossly and histologically normal. Histological examination of the solid part of broad ligament tumor, closely next to the fallopian tube, revealed a serous adenocarcinoma. Immunohistochemically, the tumor cells were strongly positive for CK7, WT-1, estrogen receptor, AE1/AE3 and EMA, and negative for CK20, D2-40 and calretinin. Also, they were negative for progesterone receptor and $\mathrm{p} 53$. The authors diagnosed the primary tumor as being a serous cystadenocarcinoma of the broad ligament [pTIC3NxM0, as modified and adapted to post-surgical staging of ovarian cancer (FIGO 2014)]. The patient has been receiving 6 cycles of adjuvant chemotherapies with one course with paclitaxel (PTX) and carboplatin (CBDCA) and five with PTX, CBDCA and Bevacizumab, and has no signs of recurrence and metastasis six months after the operation.
\end{abstract}




\section{Keywords}

Primary Broad Ligament Cancer, Serous Cystadenocarcinoma, Immunohistochemistry, Mullerian Tumor

\section{Introduction}

Primary tumors in the uterine broad ligament are rare [1], especially malignant tumors, either epithelial or non-epithelial [1] [2] [3] [4] [5], although the secondary involvement of the broad ligament by the malignant tumor is common. The developmental origin of some broad ligament tumors was described to be Wolffian origin, meanwhile others identified as having a Mullerian origin on their immunohistochemical and histologic features [6] [7]. Malignant tumors of the broad ligament have been extremely rare and only under 30 cases have been reported [8] [9], thus their epidemiology, pathogenesis, standard care/treatment, follow-up strategy and prognoses are still uncertain. The authors report here a case of primary serous cystadenocarcinoma in an 86-year-old woman, with cytological and immunohistochemical findings.

\section{Case Report}

An 86-year-old Japanese woman, gravida 3 para 3, consulted the Department of Obstetrics and Gynecology and was referred to the Department of Urology at Gujo City Hospital because of the huge pelvic mass. The patient had undergone inserting a bladder indwelling catheter for three months. The patient had received an artificial hip joint replacement before, but had no other medical histories. Magnetic resonance imaging (MRI) showed a huge pelvic mass, suggesting left ovarian cystic tumor (Figure 1(a), Figure 1(b)). Tumor markers, including CA125 (16.1 U/ml), CA19-9 $(12.8 \mathrm{U} / \mathrm{ml})$, and CA72-4 $(3.1 \mathrm{U} / \mathrm{ml})$, were within the reference values (CA125, 0 - $35.0 \mathrm{U} / \mathrm{ml}$; CA19-9, 0 - $37.0 \mathrm{U} / \mathrm{ml}$; and CA72-4, $0-10.0 \mathrm{U} / \mathrm{ml}$ ). The patient hoped for a laparoscopic surgery because of her elder age. An examining laparoscopy was started for removing the pelvic tumor. At laparoscopy, the huge pelvic mass was found within the left broad ligament (Figure 2(a)). Both ovaries, fallopian tubes and uterus were almost normal in appearance. Other disseminated tumors were also not found in the pelvic cavity. Thus, the tumor was removed together by total laparoscopic hysterectomy and bilateral salpingo-oophorectomies after the suction of serous content $(900 \mathrm{ml})$ in the broad ligament (Figure 2(b)). A small amount of ascites was also aspirated. Cytological examinations of the tumor content, ascites (Figure 3), and tumor imprint smear during operation were performed. Their findings were almost similar and suggested serous adenocarcinoma. The patient could urinate by herself after the operation.

The resected both ovaries and fallopian tubes were grossly and histologically normal. After sucking the contents, the cystic tumor of the left broad ligament 

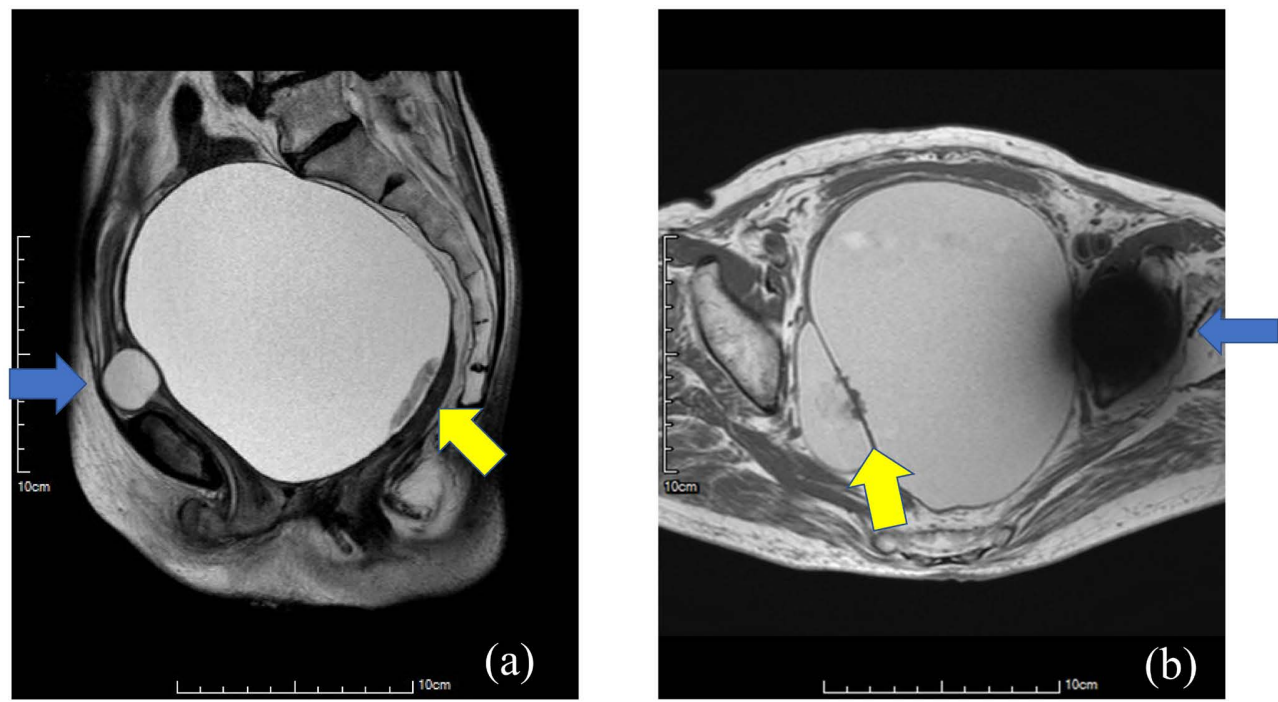

Figure 1. (a) A sagittal section in T2-weighted MRI reveals a huge cystic pelvic mass, approximately $14 \mathrm{~cm}$ in diameter, possesses a solid part (yellow arrow). A blue arrow shows urinary bladder indwelling balloon catheter. (b) A horizontal section in T2-weighted MRI indicates a solid lesion between two cystic lesions (yellow arrow). A blue arrow shows an artifact shadow due to an artificial hip joint replacement.
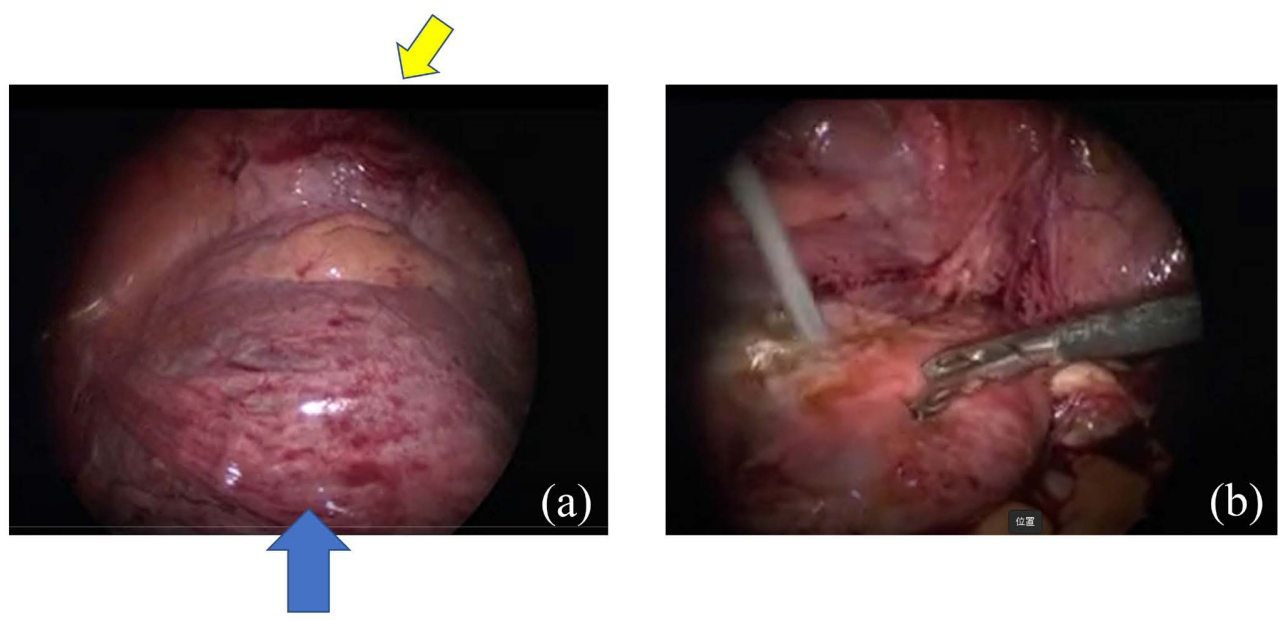

Figure 2. Laparoscopic images during the operation. (a) A huge cystic mass (blue arrow) is present at the cranial side of the uterine cervix (yellow arrow). (b) The serous content was sucked from the cystic tumor through a suction pipe.

was present (Figure 4(a), Figure 4(b)). Histological examination of the solid part of broad ligament tumor, closely next to the fallopian tube, revealed a serous cystadenocarcinoma (moderately differentiated) with numerous psammoma bodies (Figure 5(a), Figure 5(b)). Immunohistochemistry was done using nine different antibodies, such as CK7 (DAKO, 1:50 dilution), CK20 (Dako, 1:250 dilution), WT-1 (Novocastra, 1:40 dilution), estrogen receptor (ER, DAKO, 1:100 dilution), progesterone receptor (PgR, DAKO, 1:100 dilution), D2-40 (Signet lab, 1:200), calretinin (Zymed, 1:2000), AE1/AE3 ((DAKO, 1:50 dilution), p53 (Dako, 1:100) and EMA (Dako, 1:100). Immunohistochemically, the tumor cells 


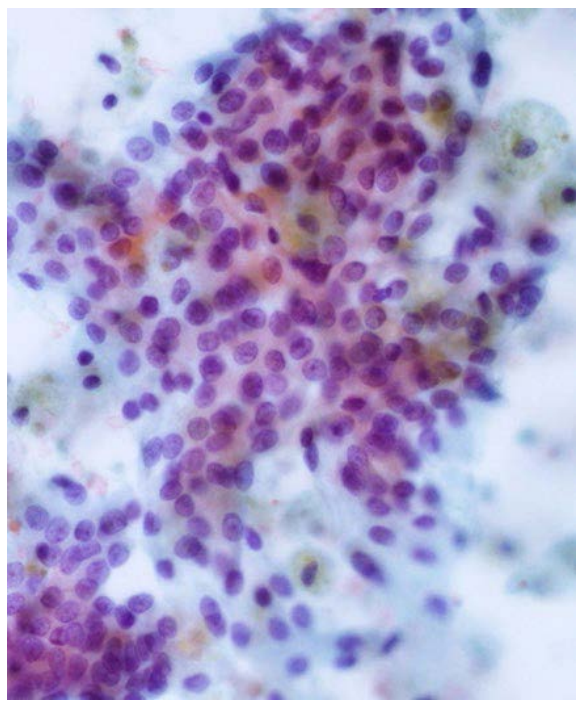

Figure 3. Cytology of the ascites. Note a large cell cluster consisted of small neoplastic cells with dense and hyperchromatic nuclei suggestive of serous adenocarcinoma. Cancer cells do not produce mucin. Papanicolaou stain, $\times 100$.
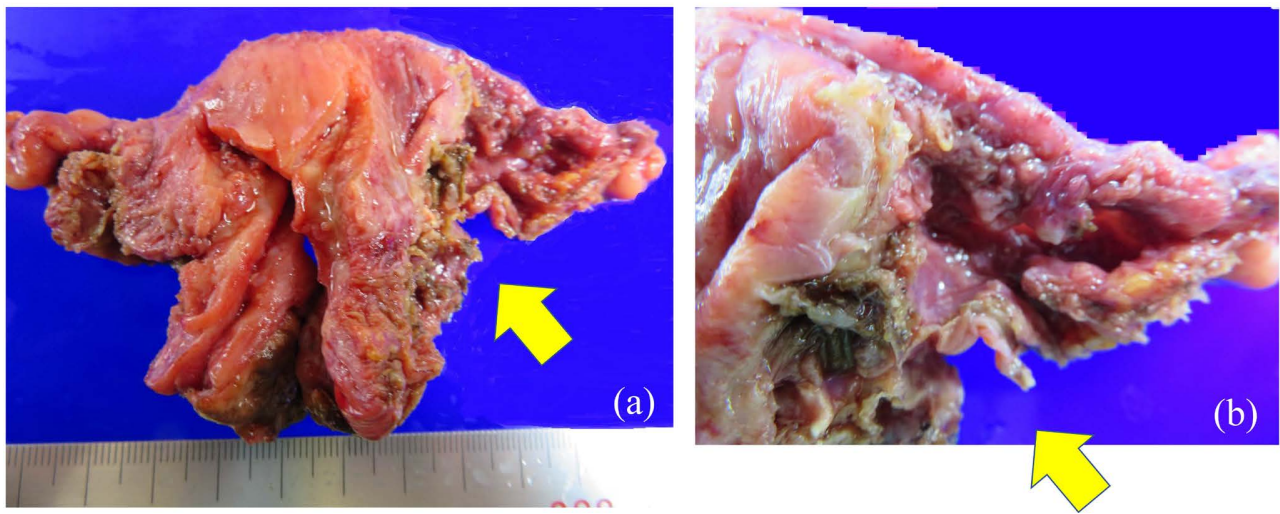

Figure 4. (a) Macroscopic view of the resected tumor arising within the left broad ligament after sucking the serous content (yellow arrow). (b) The cystic lesion is able to be separated from the left tube and ovary (yellow arrow).
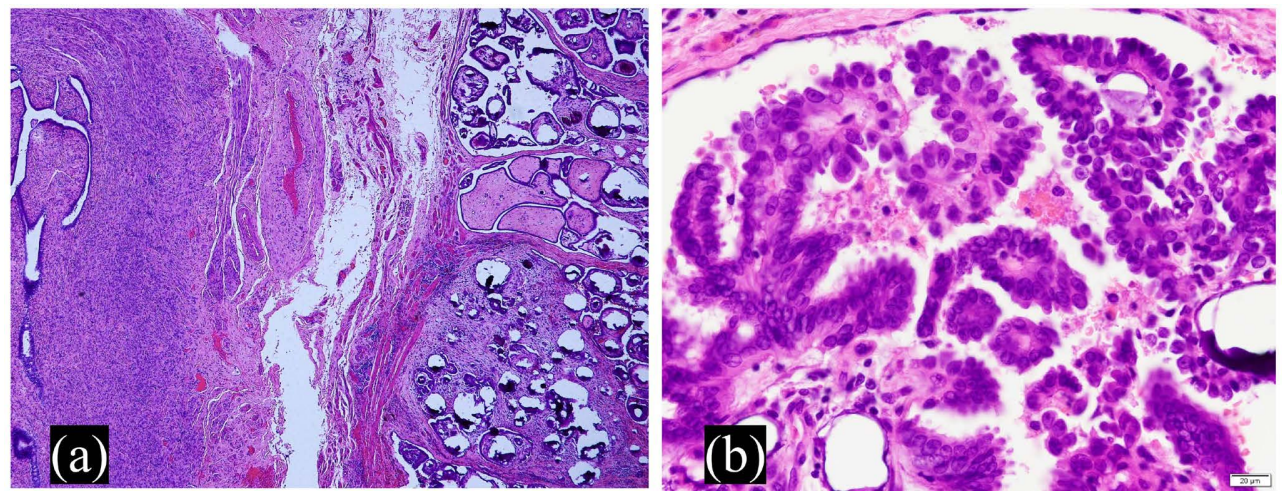

Figure 5. Microscopic views of the resected tumor. (a) The solid part of the left broad ligament tumor (right) and fallopian tube (left) are separated. The tumor shows glandular structures with many psammoma bodies. (b) Neoplastic cells show papillary structures with slight nuclear atypia. $\mathrm{H} \&$ E stain, (a) $\times 50$ and (b) $\times 200$. 
were strongly positive for CK7 (Figure 6(a)), WT-1 (Figure 6(c)), ER (Figure 6(d)), AE1/AE3 and EMA (Figure 6(f)), and negative for CK20 (Figure 6(b)), D2-40 and calretinin. Also, they were negative for PgR (Figure 6(e)) and p53. By detailed examination of the fallopian tubes, as a possible origin of serous adenocarcinoma, we could not identify any intraepithelial carcinoma lesions of the fimbria. The authors diagnosed the primary tumor as being a serous cystadenocarcinoma, moderately differentiated of the broad ligament [pTIC3NxM0, as modified and adapted to post-surgical staging of ovarian cancer (FIGO 2014)]. The patient has been receiving 6 cycles of adjuvant chemotherapies with one course with paclitaxel (PTX) and carboplatin (CBDCA) and five with PTX, CBDCA and Bevacizumab, and has no signs of recurrence and metastasis six months after the operation.

To assess the poly(ADP-ribose) polymerase (PARP) inhibitor sensitivity in the present case, homologous recombination deficiency (HRD) by assessing genomic instability score (GIS) and BRCA1/2 mutational status in genomic DNA extracted from tumor specimens were analyzed by Myriad MyChoice system $^{\circledR}$ (Myriad Genetic Laboratories, Inc. Utah) [10]. As a result, GIS was unable to be determined, and tumor mutation $B R C A 1 / 2$ status was negative for a clinically significant mutation. Thus, the patient is not sensitive for PARP inhibitor (Olaparib $^{\circledR}$ ) for a maintenance treatment [11].

\section{Discussion}

It is considered to be very difficult to properly diagnose a tumor of the broad ligament before surgery, because of their rarity and anatomical occurring sites [2] [9]. Actually, our initial diagnosis of the present case was diagnosed as a cystic ovarian tumor including the solid part before surgery. An examining laparoscopy
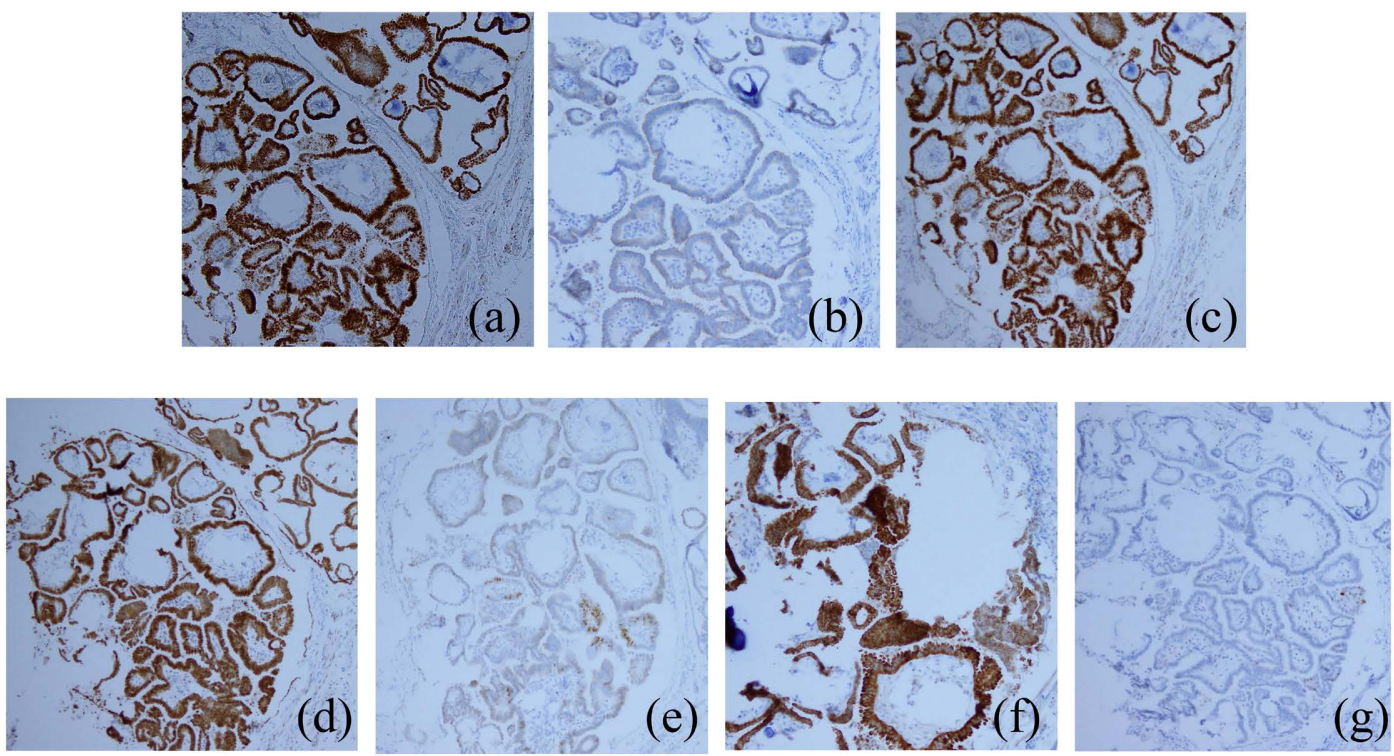

Figure 6. Immunohistochemistry of the tumor cells that are positive for (a) CK7, (c) WT-1, (d) ER, and (f) EMA, while negative for (b) CK20, (e) PgR, and (g) p53. Immunohistochemistry, (a)-(g), $\times 80$. 
was started for removing the pelvic tumor. At laparoscopy, the huge pelvic mass was found within the left broad ligament. Thus, the tumor was removed together by total laparoscopic hysterectomy and bilateral salpingo-oophorectomies after the suction of serous content in the broad ligament.

As diagnostic criteria for the primary malignant tumor of the broad ligament, Gardner et al. proposed a primary location within or on the surface of the broad ligament and a complete separation of the tumor from the uterus, ipsilateral ovary and fallopian tube [12]. When the criteria were applied, primary carcinomas of the broad ligament were extremely rare; only under 30 cases have been reported [8] [9]. In the present case, serous cystadenocarcinoma was only recognized in the solid part of the huge cystic mass of the broad ligament, and macroscopically and histologically no other lesions could be found in the both ovaries and fallopian tubes. This case fulfilled the Gardner's diagnostic criteria.

Immunohistochemical analysis of WT-1 is reported to be useful in confirming almost $90 \%$ ovarian cancer cases and is most specific with serous type cancers [13]. Immunohistochemically, CK7 positive and CK20 negative tumors were resembling ovarian serous carcinoma [14]. Thus, the immunohistochemical expression pattern resembled the serous ovarian cancer, suggested to be derived from Mullerian origin [14]. Most of the epithelial malignant tumors of the broad ligament are derived from the Mullerian remnants, including serous, mucinous, clear cell, and endometrioid types of malignancy [9]. The most common type of the broad ligament cancer is reported to be serous cystadenocarcinoma, as shown in this case [9].

Originally, serous carcinoma of the broad ligament has been suggested to occur as a tubal intra-epithelial carcinoma and then progress an invasive carcinoma [15]. Another hypothesis includes the origin of a low-grade serous carcino$\mathrm{ma}$ is via transformation of benign/borderline serous tumors, which are thought to be derived from inclusion cysts originating from the ovarian surface epithelium [15]. In addition, p53 was reported to be positive in most of serous tubal in-situ carcinoma and high grade serous ovarian carcinoma [16], while the present case was negative for $\mathrm{p} 53$. Thus, our case may be low-grade malignancy or borderline of serous tumor.

The role of postsurgical adjuvant chemotherapy against broad ligament cancer has been also controversial [9]: in half of the cases, only surgery has been performed; surgery followed by the chemotherapy in one-fourth of cases; and surgery followed by radiotherapy in $18 \%$ of cases [9]. Although the suggested treatment for a malignant broad ligament tumor has not been established, at least the Mullerian type tumor should be treated with the same strategies used for an epithelial ovarian cancer, because of similar histopathology [1]. We could not perform partial omentectomy or lymph nodes dissection that is usually done for adnexal tumor, because the examining laparoscope was started for removing the pelvic tumor. Although the clinical post-surgical stage was pTIC3NxM0, the patient received the post-surgical chemotherapy, including PTX, CBDCA and Bevaci- 
zumab, in accordance with stage III [17].

\section{Conclusion}

An 86-year-old Japanese woman underwent an examining laparoscopy for removing the huge pelvic tumor, but the tumor was present within the left broad ligament. Thus, we removed the tumor by hysterectomy and bilateral salpingo-oophorectomies under laparoscope. The resected ovaries and fallopian tubes were grossly and histologically normal. Immunohistochemically, the tumor cells were strongly positive for CK7, WT-1, ER, AE1/AE3 and EMA, and negative for CK20, D2-40, calretinin, PgR and p53. We finally diagnosed the primary tumor as being a serous cystadenocarcinoma of the broad ligament [pTIC3NxM0, as modified and adapted to post-surgical staging of ovarian cancer (FIGO 2014)]. The patient has been receiving 6 cycles of adjuvant chemotherapies with one course with PTX and CBDCA and five with PTX, CBDCA and Bevacizumab, and has been without any sign of recurrence six months after the operation.

\section{Consent}

Agreement by a document was obtained from the patient before writing this case report.

\section{Ethical Approval}

This was obtained from the ethical committee of our hospital before writing this case report (ethical approval no. 21062501).

\section{Acknowledgements}

We thank the editor and reviewers for the constructive comments, which helped us to improve the manuscript. This case report was approved by the patient verbal consent.

\section{Conflicts of Interest}

The authors declare that they have no competing interests.

\section{References}

[1] Aslani, M. and Scully, R.E. (1989) Primary Carcinoma of the Broad Ligament. Report of Four Cases and Review of the Literature. Cancer, 64, 1540-1545. https://doi.org/10.1002/1097-0142(19891001)64:7\%3C1540::AID-CNCR2820640731 \%3E3.0.CO;2-P

[2] Itani, Y., Itoh, K., Adachi, S., Koyama, M., Kimura, T., Chin, R. and Takemura, T. (2002) Malignant Epithelial Tumor of Unknown Origin of the Broad Ligament. Archives of Gynecology and Obstetrics, 267, 113-116. https://doi.org/10.1007/s00404-001-0264-5

[3] Kobayashi, Y., Yamazaki, K., Shinohara, M., Iwahashi, K., Suzuki, A., Fujii, T., Sasaki, H. and Shiraishi, S. (1996) Undifferentiated Carcinoma of the Broad Ligament in a 28-Year-Old Woman-A Case Report and Results of Immunohistochemical 
and Electron-Microscopic Studies. Gynecologic Oncology, 63, 382-387. https://doi.org/10.1006/gyno.1996.0340

[4] Loverro, G., Cormio, G., Renzulli, G., Lepera, A., Ricco, R. and Selvaggi, L. (1997) Serous Papillary Cystadenoma of Borderline Malignancy of the Broad Ligament. European Journal of Obstetrics \& Gynecology and Reproductive Biology, 74, 211-213. https://doi.org/10.1016/S0301-2115(97)00108-5

[5] Shah, A., Finn, C. and Light, A. (2003) Leiomyosarcoma of the Broad Ligament: A Case Report and Literature Review. Gynecologic Oncology, 90, 450-452. https://doi.org/10.1016/S0090-8258(03)00208-7

[6] Czernobilsky, B. and Lancet, M. (1972) Broad Ligament Adenocarcinoma of Müllerian Origin. Obstetrics \& Gynecology, 40, 238-242.

[7] Rojansky, N., Ophir, E., Sharony, A., Spira, H. and Suprun, H. (1985) Broad Ligament Adenocarcinoma-Its Origin and Clinical Behavior. A Literature Review and Report of a Case. Obstetrical \& Gynecological Survey, 40, 665-671. https://doi.org/10.1097/00006254-198511000-00001

[8] Elangovan, A., Dracham, C.B., Muddabhaktuni, M.C. B. and Zaidi, A. (2020) Primary Broad Ligament Adenocarcinoma. Autopsy and Case Reports, 10, Article ID: e2020176. https://doi.org/10.4322/acr.2020.176

[9] Miyoshi, A., Miyatake, T., Hara, T., Komiya, S., Komura, N., Tanaka, A., Kanao, S., Takeda, M., Mimura, M., Nagamatsu, M., Yamasaki, M. and Yokoi, T. (2016) Rare Primary Adenocarcinoma of the Broad Ligament: Report of Two Cases and a Literature Review. International Journal of Surgical Pathology, 24, 436-442.

https://doi.org/10.1177/1066896915622690

[10] Telli, M.L., Timms, K.M., Reid, J., Hennessy, B., Mills, G.B., Jensen, K.C., Szallasi, Z., Barry, W.T., Winer, E.P., Tung, N.M., Isakoff, S.J., Ryan, P.D., Greene-Colozzi, A., Gutin, A., Sangale, Z., Iliev, D., Neff, C., Abkevich, V., Jones, J.T., Lanchbury, J.S., Hartman, A.R., Garber, J.E., Ford, J.M., Silver, D.P. and Richardson, A.L. (2016) Homologous Recombination Deficiency (HRD) Score Predicts Response to Platinum-Containing Neoadjuvant Chemotherapy in Patients with Triple-Negative Breast Cancer. Clinical Cancer Research, 22, 3764-3773. https://doi.org/10.1158/1078-0432.CCR-15-2477

[11] Moore, K.N., Secord, A.A., Geller, M.A., Miller, D.S., Cloven, N., Fleming, G.F., Wahner Hendrickson, A.E., Azodi, M., Di Silvestro, P., Oza, A.M., Cristea, M., Berek, J.S., Chan, J.K., Rimel, B.J., Matei, D.E., Li, Y., Sun, K., Luptakova, K., Matulonis, U.A. and Monk, B.J. (2019) Niraparib Monotherapy for Late-Line Treatment of Ovarian Cancer (QUADRA): A Multicentre, Open-Label, Single-Arm, Phase 2 Trial. Lancet Oncology, 20, 636-648. https://doi.org/10.1016/S1470-2045(19)30029-4

[12] Gardner, G.H., Greene, R.R. and Peckham, B. (1957) Tumors of the Broad Ligament. American Journal of Obstetrics and Gynecology, 73, 536-555.

https://doi.org/10.1016/S0002-9378(16)37427-0

[13] Lerwill, M.F. (2004) Current Practical Applications of Diagnostic Immunohistochemistry in Breast Pathology. American Journal of Surgical Pathology, 28, 1076-1091. https://doi.org/10.1097/01.pas.0000126780.10029.f0

[14] Cathro, H.P. and Stoler, M.H. (2002) Expression of Cytokeratins 7 and 20 in Ovarian Neoplasia. American Journal of Clinical Pathology, 117, 944-951. https://doi.org/10.1309/2T1Y-7BB7-DAPE-PQ6L

[15] Handa, Y., Kato, H., Kaneuchi, M., Saitoh, Y. and Yamashita, K. (2007) High-Grade Broad Ligament Cancer of Mullerian Origin: Immunohistochemical Analysis of a Case and Review of the Literature. International Journal of Gynecological Cancer, 
17, 705-709.

[16] Lim, D. and Oliva, E. (2013) Precursors and Pathogenesis of Ovarian Carcinoma. Pathology, 45, 229-242. https://doi.org/10.1097/PAT.0b013e32835f2264

[17] Leonhardt, K., Einenkel, J., Sohr, S., Engeland, K. and Horn, L.C. (2011) p53 Signature and Serous Tubal In-Situ Carcinoma in Cases of Primary Tubal and Peritoneal Carcinomas and Serous Borderline Tumors of the Ovary. International Journal of Gynecological Pathology, 30, 417-424.

https://doi.org/10.1097/PGP.0b013e318216d447 